\title{
The Impact of Nail Psoriasis and Treatment on Quality of Life: A Systematic Review
}

\author{
Claire R. Stewart ${ }^{a} \quad$ Leah Algu $^{\text {b }}$ Rakhshan Kamran ${ }^{c}$ Cameron F. Leveille ${ }^{d}$ \\ Khizar Abid $^{\mathrm{b}}$ Charlene Rae $^{\mathrm{e}}$ Shari R. Lipner ${ }^{\mathrm{f}}$
}

aWeill Cornell Medical College, New York, NY, USA; baculty of Health Sciences, McMaster University, Hamilton, ON,

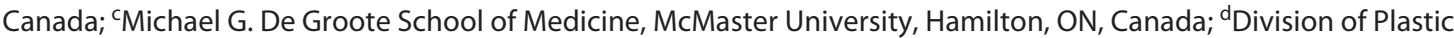
Surgery, Department of Surgery, McMaster University, Hamilton, ON, Canada; 'Department of Pediatrics, McMaster University, Hamilton, ON, Canada; fDepartment of Dermatology, Weill Cornell Medical College, New York, NY, USA

\section{Keywords}

Nail psoriasis · Quality of life · Systematic review · Indicators . Nail Psoriasis Severity Index. Psoriasis Area Severity Index . Dermatology Life Quality Index. Patient-reported outcome measures

\begin{abstract}
At least $80 \%$ of patients with psoriasis will have nail involvement during their lifetimes. Understanding quality of life (QoL) impact of this condition and associated treatments is of utmost importance. Study objectives were to review the available literature describing patient-reported QoL outcomes in nail psoriasis and relationship with disease severity and treatment. A literature search was performed for English-language articles published prior to August 1, 2020. Articles were included in the review if primary data and validated patient-reported outcome measures assessing QoL were presented, and nail involvement was specifically examined. Fifteen studies were included in the final analysis. Patients with nail psoriasis had higher Psoriasis Area Severity Index and Dermatology Life Quality Index scores than those with psoriasis without nail involvement. The largest percent
\end{abstract}

karger@karger.com www.karger.com/sad

Karger ${ }^{\prime \prime} \div$

GOPEN ACCESS
(C) 2021 The Author(s)

Published by S. Karger AG, Basel

This is an Open Access article licensed under the Creative Commons Attribution-NonCommercial-4.0 International License (CC BY-NC) (http://www.karger.com/Services/OpenAccessLicense), applicable to the online version of the article only. Usage and distribution for commercial purposes requires written permission. improvement in QoL score was associated with adalimumab. Studies investigating topicals, intralesionals, and systemic treatments were excluded since only biologic studies utilized validated patient-reported outcome measures. This review affirms that nail psoriasis is physically and emotionally distressing, warranting prompt treatment. Increased efforts are needed to address the impact of treatment on patient QoL using validated outcome measures that assess cosmetic, physical, and social problems.

(C) 2021 The Author(s)

Published by S. Karger AG, Basel

\section{Introduction}

Psoriasis is a chronic skin disorder affecting about 2\% of the US population [1]. At least $80 \%$ of psoriasis patients will have nail involvement in their lifetimes, while an estimated $10 \%$ have isolated nail psoriasis [1]. Clinical findings include nail plate pitting, onycholysis, splinter hemorrhages, "oil spots," and subungual hyperkeratosis. Yet the impact of nail psoriasis extends beyond aesthetics, as this condition causes pain, functional impairment, and embarrassment. 
Fig. 1. Flow diagram of the study selection process.

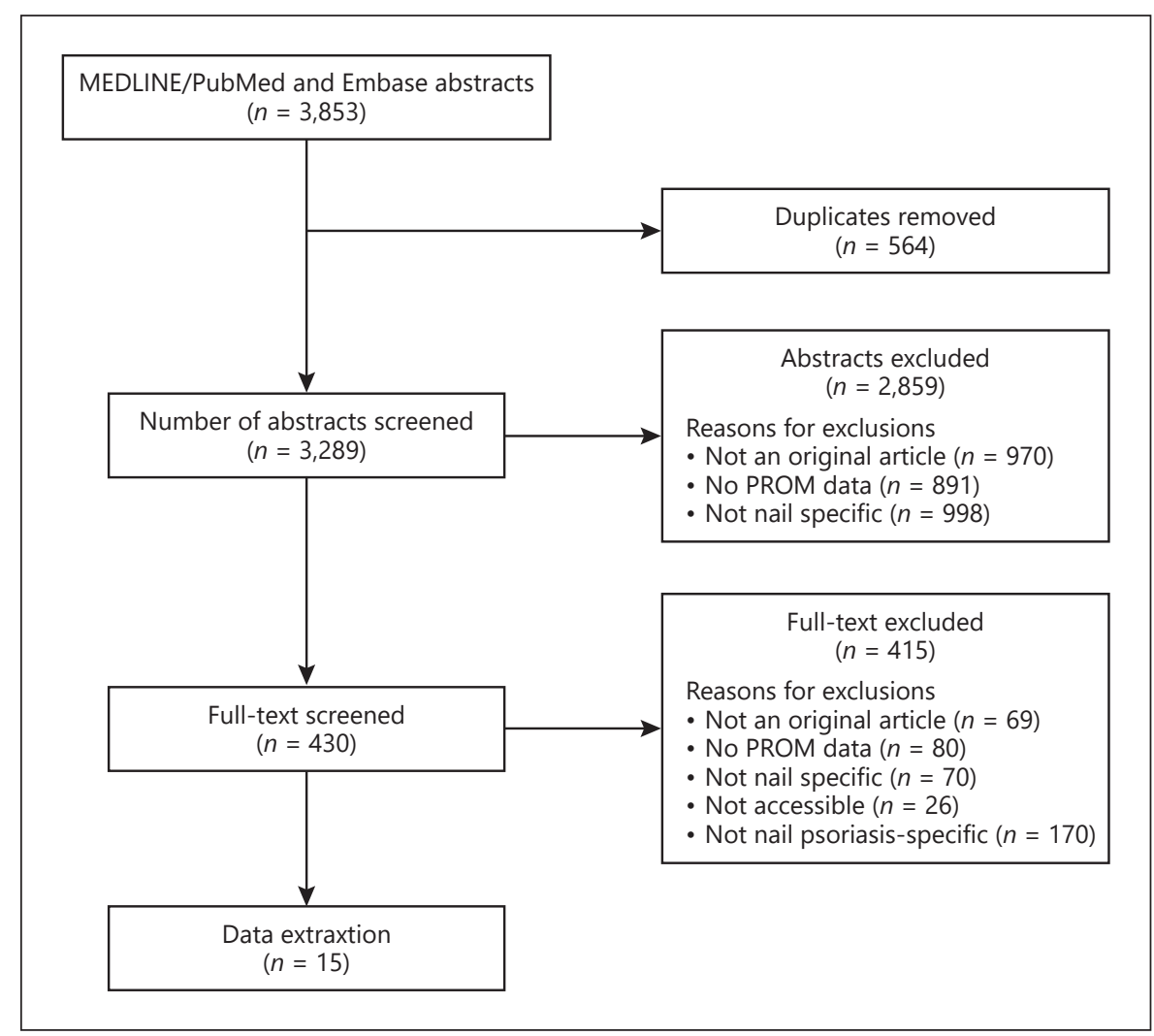

While there is an expanding number of treatments for nail psoriasis, patient comorbidities, medication interactions, potential adverse effects, and costs must be considered [2]. Therefore, it is crucial to understand the impact of nail psoriasis on quality of life (QoL) to recommend treatment. Patient-reported outcome measures (PROMs) are useful tools to help patients vocalize their concerns. This review sought to evaluate the available literature describing the relationship of psoriasis severity and treatment with QoL outcomes as assessed by PROMs in nail psoriasis.

\section{Materials and Methods}

A search of the English-language literature published prior to August 1, 2020, for studies reporting QoL in patients with nail psoriasis was performed. MEDLINE and Embase databases were examined with the search terms "nail" and "QoL" (C.F.L). Abstracts were screened by 2 researchers (R.K. and L.A.) using the exclusion criteria: not an original article, valid PROM not used, and outcomes not reported in patient subgroup with nail involvement. Full-text articles were reviewed by 2 researchers (R.K. and L.A.) with discrepancies resolved by a third (C.R.). References of articles were searched to identify additional articles that may have been missed, although no studies were added (C.F.L.). Data were ex- tracted and confirmed by 2 researchers (C.S. and L.A.). Study design, population demographics, severity, and QoL scores were extracted. To measure evidence level, we used a 5-point scale with the following ratings: (1) randomized clinical trial or systematic review with meta-analysis; (2) controlled trial without randomization or prospective cohort trial; (3) case-control study or retrospective cohort study; (4) cross-sectional study or case series with more than 5 patients; and (5) case report.

\section{Results}

A total of 430 full-text articles were assessed for eligibility with 15 studies included in this analysis. The study selection process is detailed in Figure 1.

\section{Patient-Reported Outcome Measures}

Five PROMS were used to assess QoL, with Dermatology Life Quality Index (DLQI) most commonly used $(n=10)$. DLQI scores correlate as $0-1$ no effect, $2-5$ small effect, 6-10 moderate effect, 11-20 large effect, and 21-30 extremely large effect [3]. The minimal clinically important difference has been established as 4.0 [4]. The International Onychomycosis-Specific Questionnaire, while 
designed to measure QoL in patients with onychomycosis, was used in 3 studies. This PROM is scored on a $0-100$ scale with 100 representing the worst QoL [5]. The Nail Psoriasis QoL measure, Nail Assessment in Psoriasis and Psoriatic Arthritis Questionnaire (NAPPA-QoL), and NPQ10 Score were used in 1 study each. In the Nail Psoriasis QoL measure, scores range from 0 to 10 with 10 as most severe impact [6]. The NAPPA-QoL is a 20 -item nail-specific QoL questionnaire assessing nail status, stigma, emotional, and everyday life impact [7]. The NPQ10 score is a nail-specific scale with scores ranging from 0 to 20 with higher scores indicating poorer QoL [8].

\section{Demographics}

The demographics of study populations were varied. The mean age of subjects across studies was between 43.3 and 57.5 years $[6,9-22]$. Composition of females in study samples also varied from 19.2 to $63.0 \%$ [6, 9-22]. The mean duration of psoriasis in those with nail involvement ranged from 11.5 to 34.3 years [6,9-22]. No studies included patients with comorbid onychomycosis.

\section{Psoriasis Severity and Quality of Life}

Eight studies compared clinician-assessed severity and DLQI in patients with and without nail involvement (Table 1) [9-15]. Severity was reported by Psoriasis Area Severity Index (PASI) and Nail Psoriasis Severity Index (NAPSI). Across studies, psoriasis with nail involvement was associated with significantly higher baseline PASI scores [9-15]. Nail involvement was also associated with significantly greater QoL impairment [9-15]. In studies with subgroup analysis by gender, women had higher PASI and DLQI scores than men $[10,12,13]$.

Reich and Szepietowski [23] $(n=30)$ found that patients with nail psoriasis were most concerned with appearance and disease worsening; NailQoL scores were correlated with NAPSI in women, but not in men. Ortonne et al. [8] $(n=795)$ reported that female gender, shorter disease duration, and presence of both fingernail and toenail involvement were associated with worse QoL, as measured by the NPQ10 Scale; PASI and NAPSI scores were not reported. In addition, $86 \%$ of patients considered nail psoriasis to be bothersome, $87 \%$ as unsightly, and $59 \%$ as painful [8].

\section{Treatment, Severity, and Quality of Life}

Nine studies reported QoL data in combination with systemic biologic treatments (Table 2) [6, 9, 16-22]. Two randomized controlled trials received the highest Level 1 quality of evidence ranking; all other prospective, openlabel studies received Level $2[6,22]$.

The Impact of Nail Psoriasis and

Treatment on Quality of Life
For all treatment modalities (infliximab, etanercept, adalimumab, ustekinumab, and secukinumab), authors reported statistically significant improvement in both NAPSI and QoL with treatment $[6,9,16-22]$. Ortonne et al. [8] reported that $72 \%$ of patients were dissatisfied with treatment, but specific therapies were not specified. Infliximab (5 mg/kg infusion at baseline, 2, 6, and every 8 weeks thereafter for 38 weeks) was associated with the greatest reported percentage improvement in NAPSI (94.1\%) [16] which was 4.3 percentage points higher than the next highest scoring treatment, ustekinumab [21], and 48.8 percentage points higher than the lowest scoring treatment, secukinumab [22]. The ustekinumab dose was $45 \mathrm{mg}$ for patients $<100 \mathrm{~kg}$ and $90 \mathrm{mg}$ for patients over $100 \mathrm{~kg}$ subcutaneous at baseline, 4,16 , and every 12 weeks thereafter for 40 weeks; secukinumab was dosed 300 or $150 \mathrm{mg}$ subcutaneous weekly for 5 weeks and every 4-16 weeks [21].

While different parameters were used to assess QoL impact, multiple studies identified similar themes in specific domains of QoL that improved with treatment. Commonly mentioned themes across medications included improvement in symptoms, pain, functionality, and cosmetic appearance of nails, as well as improvements in personal relationships, business interactions, and ability to perform daily activities [16-18, 22].

When comparing reported percent improvement in QoL across treatment types, adalimumab had the greatest percent improvement from baseline. In 1 study, there was a $87.7 \%$ improvement in DLQI score, and in another study, a $88.2 \%$ improvement in Nail Psoriasis QoL Score $[6,19]$. Of note, in a recent study by Kokolakis et al. [20], there was a lower percent improvement than Khobzey et al. [19] (60.0 vs. 88.2\%) in DLQI. This difference may be due to the longer duration of disease in the study population investigated by Kokolakis et al. [19, 20]. In the study, using the Onychomycosis-Specific Questionnaire, the authors reported a mean improvement of $74.4 \%$ from baseline, while the studies reporting Nail Psoriasis QoL found $87.7 \%$ improvement $[6,18]$. The percentage of patients with concomitant joint involvement ranged from 28.6 to $66.7 \%$ in these studies [6, 1820].

\section{Discussion}

This review affirms that nail psoriasis is associated with both worse clinician-assessed severity and larger patient-reported QoL impact compared to psoriasis pa-

Skin Appendage Disord 2021;7:83-89 


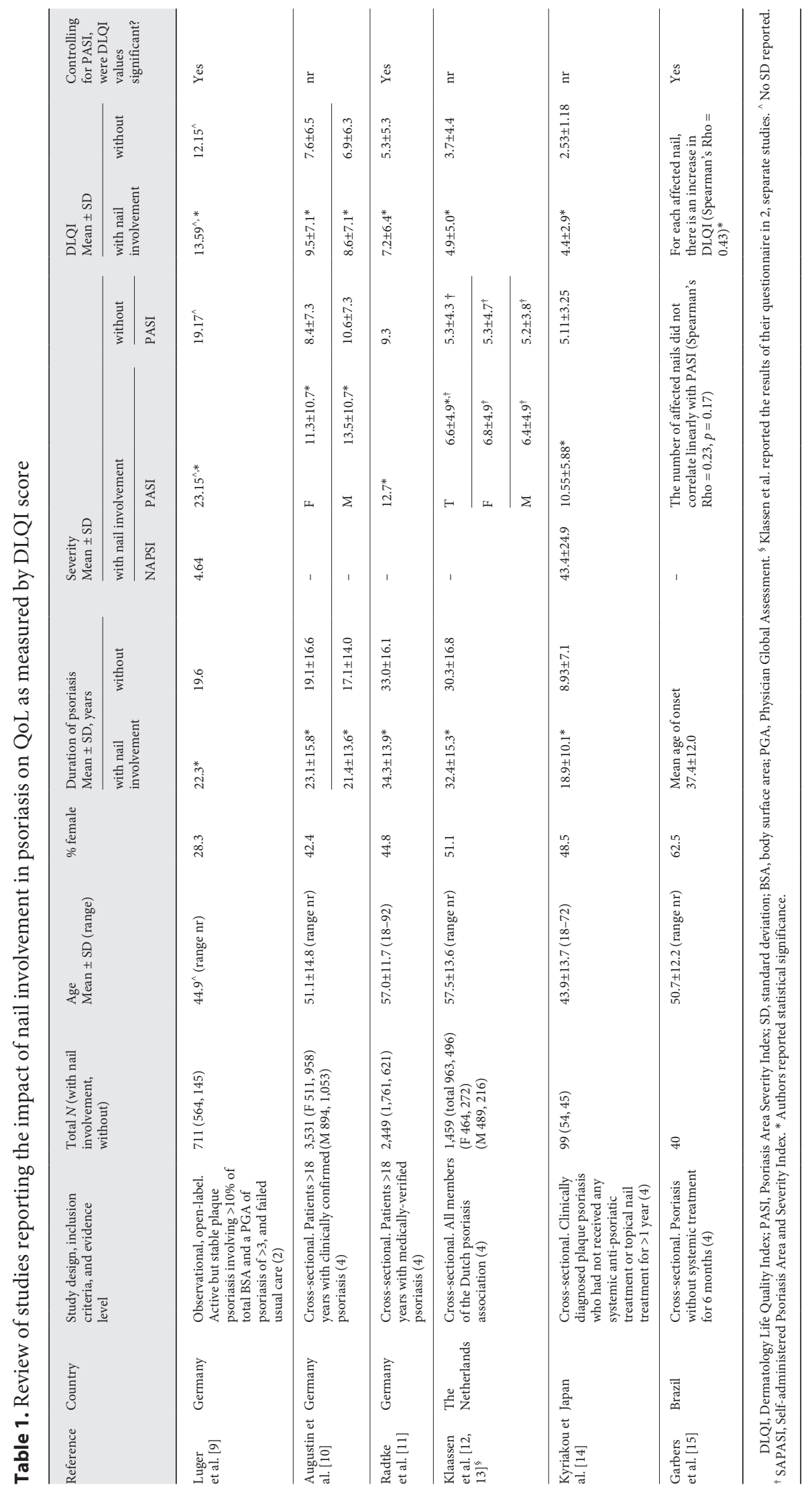




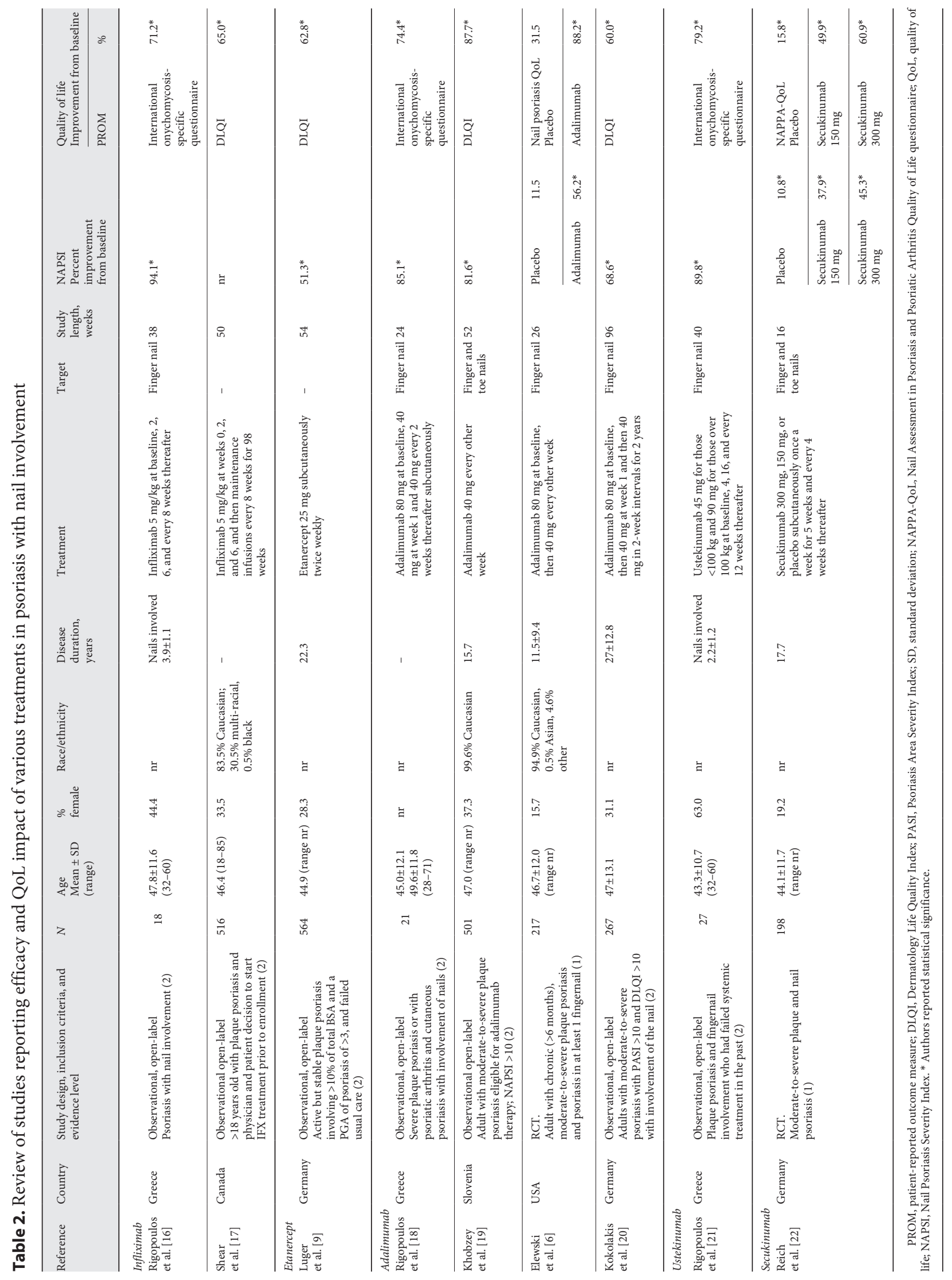


tients without nail involvement. Furthermore, the reviewed studies confirm that treatment of nail psoriasis leads to improved QoL. In our review, adalimumab was associated with the greatest percentage improvement in health-related QoL.

Some clinical trials have incorporated PROMs, however, analyzing patient QoL with validated outcome measures should be standard [24]. Our study also raised concerns as to whether existing PROMs can adequately evaluate the impact of nail changes. Though all studies found that DLQI is higher in patients with nail involvement, the differences in scores ranged from 1.2 to 1.9, which is below the minimal clinically important difference [4]. These findings contrast with clinical practice, in which patients with nail disorders have aesthetic, physical, and social disabilities. The DLQI, which is designed for general skin conditions, may not have relevant content validity to measure the outcomes most important in nail psoriasis. In a review of health-related QoL in patients with nail disorders, Reich and Szepietowski [23] pointed out that when DLQI is applied to patients with both skin and nail involvement, the instrument cannot differentiate between the influence of nail versus skin changes on QoL. Since no studies excluded patients with psoriatic arthritis, it is not clear whether improvement in QoL scores was attributable to improvement in nail or joint symptoms. Only 3 studies utilized nail psoriasis-specific PROMs, thus limiting our comparisons.

This study is subject to several limitations. The lack of literature evaluating QoL and meeting our screening criteria limits the generalizability of our findings. None of the studies included children and only the studies by Radtke et al. [11] and Shear et al. [17] included adults over the age of 75 , despite nail psoriasis being a concern in both pediatric and older patients. In the Joint American Academy of Dermatology - National Psoriasis Foundation guidelines for the treatment of psoriasis in pediatric patients, Menter et al. [25] recommended for consideration of the impact of disease on the "physical, social, and psychological QoL and/or activities of daily living" when determining the severity of psoriasis in children. Additionally, they highlighted the use of the Children's DLQI, a derivative of the DLQI designed for patients aged 4- to 11 -year old - a recommendation that we support for future research in this subgroup $[25,26]$. Another limitation is that topicals, intralesionals, and systemics were excluded; only studies investigating biologic therapies met inclusion criteria for our review. Additionally, only 2 studies reported whether patients had previous exposure to biologics, though others did indicate that there was a washout period $[17,22]$. If patients had failed treatment with other biologics, they may have had more severe disease with poorer QoL. The strength of evidence for the treatment studies was quite high, and the risk of bias was deemed to be low.

\section{Conclusions}

This review confirms that nail psoriasis is physically and emotionally distressing, warranting prompt and adequate treatment. However, the paucity of data limited types of treatments included in our analysis highlight the necessity of further research. While future studies should include PROMs as metrics to incorporate the patient perspective of the social, emotional, and functional impact of nail psoriasis, it is crucial that these PROMs capture the aspects most important to the patient and have content validity for use in nail psoriasis.

\section{Statement of Ethics}

Ethics approval was not required for this systematic review because all data was publicly available.

\section{Conflict of Interest Statement}

The authors have no conflicts of interest to disclose.

\section{Funding Sources}

The authors did not receive any funding.

\section{Author Contributions}

Claire Stewart was responsible for data extraction, writing of the manuscript, and figure creation. Leah Algu screened abstract and full-text articles and conducted data extraction. Rakhshan $\mathrm{Ka}$ mran screened abstract and full-text articles. Cameron Leveille conducted publication search and organized search results. Khizar Abid found and organized full-text articles. Charlene Rae oversaw coordination and created the data extraction sheet. Shari Lipner is the senior author who conceived the study and wrote the manuscript. All authors reviewed the manuscript.
Stewart/Algu/Kamran/Leveille/Abid/Rae/ Lipner 


\section{References}

1 Jiaravuthisan MM, Sasseville D, Vender RB, Murphy F, Muhn CY. Psoriasis of the nail: anatomy, pathology, clinical presentation, and a review of the literature on therapy. J Am Acad Dermatol. 2007;57(1):1-27.

2 Rigopoulos D, Baran R, Chiheb S, Daniel CR, Di Chiacchio N, Gregoriou S, et al. Recommendations for the definition, evaluation, and treatment of nail psoriasis in adult patients with no or mild skin psoriasis: a dermatologist and nail expert group consensus. J Am Acad Dermatol. 2019 Jul;81(1):228-40.

3 Finlay AY, Khan GK. Dermatology Life Quality Index (DLQI): a simple practical measure for routine clinical use. Clin Exp Dermatol. 1994 May;19(3):210-6.

4 Basra MK, Salek MS, Camilleri L, Sturkey R, Finlay AY. Determining the minimal clinically important difference and responsiveness of the Dermatology Life Quality Index (DLQI): further data. Dermatology. 2015; 230(1):27-33.

5 Drake LA, Patrick DL, Fleckman P, Andr J, Baran R, Haneke E, et al. The impact of onychomycosis on quality of life: development of an international onychomycosis-specific questionnaire to measure patient quality of life. J Am Acad Dermatol. 1999 Aug;41(2 Pt 1):189-96.

6 Elewski BE, Okun MM, Papp K, Baker CS, Crowley JJ, Guillet G, et al. Adalimumab for nail psoriasis: efficacy and safety from the first 26 weeks of a phase 3 , randomized, placebocontrolled trial. J Am Acad Dermatol. 2018 Jan;78(1):90-e1.

7 Augustin M, Blome C, Costanzo A, Dauden E, Ferrandiz C, Girolomoni G, et al. Nail Assessment in Psoriasis and Psoriatic Arthritis (NAPPA): development and validation of a tool for assessment of nail psoriasis outcomes. Br J Dermatol. 2014 Mar;170(3):591-8.

8 Ortonne JP, Baran R, Corvest M, Schmitt C, Voisard JJ, Taieb C. Development and validation of nail psoriasis quality of life scale (NPQ10). J Eur Acad Dermatol Venereol. 2010 Jan;24(1):22-7.
9 Luger TA, Barker J, Lambert J, Yang S, Robertson D, Foehl J, et al. Sustained improvement in joint pain and nail symptoms with etanercept therapy in patients with moderateto-severe psoriasis. J Eur Acad Dermatol Venereol. 2009 Aug;23(8):896-904.

10 Augustin M, Reich K, Blome C, Schäfer I, Laass A, Radtke MA. Nail psoriasis in Germany: epidemiology and burden of disease. Br J Dermatol. 2010 Sep;163(3):580-5.

11 Radtke MA, Langenbruch AK, Schäfer I, Herberger K, Reich K, Augustin M. Nail psoriasis as a severity indicator: results from the PsoReal study. Patient Relat Outcome Meas. 2011 Jul;2:1-6.

12 Klaassen KM, van de Kerkhof PC, Pasch MC. Nail psoriasis: a questionnaire-based survey. Br J Dermatol. 2013 Aug;169(2):314-9.

13 Klaassen KM, van de Kerkhof PC, Pasch MC. Nail psoriasis, the unknown burden of disease. J Eur Acad Dermatol Venereol. 2014 Dec;28(12):1690-5.

14 Kyriakou A, Patsatsi A, Sotiriadis D. Quality of life and severity of skin and nail involvement in patients with plaque psoriasis. Eur J Dermatol. 2014 Sep-Oct;24(5):623-5.

15 Garbers LE, Slongo H, Fabricio LH, Schmitt JV, Bonalumi A. Incidence, clinical manifestations and clipping of nail psoriasis in the dermatology center of the Hospital Universitário Evangélico de Curitiba. An Bras Dermatol. 2016 May-Jun;91(3):300-5.

16 Rigopoulos D, Gregoriou S, Stratigos A, Larios G, Korfitis C, Papaioannou D, et al. Evaluation of the efficacy and safety of infliximab on psoriatic nails: an unblinded, nonrandomized open-label study. Br J Dermatol. 2008 Aug;159(2):453-6.

17 Shear NH, Hartmann M, Toledo-Bahena ME, Gilbert M, Katsambas A, Yao R, et al. Healthrelated quality-of-life improvements during 98 weeks of infliximab therapy in patients with plaque-type psoriasis in real-world practice. Qual Life Res. 2016 Aug;25(8):2031-40.

18 Rigopoulos D, Gregoriou S, Lazaridou E, Belyayeva E, Apalla Z, Makris M, et al. Treatment of nail psoriasis with adalimumab: an open label unblinded study. J Eur Acad Dermatol Venereol. 2010 May;24(5):530-4.
19 Khobzey K, Liskova I, Szegedi A, Pavlovsky L, Lunder T, Kingo $\mathrm{K}$, et al. Effectiveness of adalimumab in the treatment of scalp and nail affection in patients with moderate to severe plaque psoriasis in routine clinical practice. Acta Dermatovenerol Alp Pannonica Adriat. 2017 Mar;26(1):11-4.

20 Kokolakis G, Bachmann F, Wolk K, Sabat R Philipp S. Efficacy of adalimumab for nail psoriasis during 24 months of continuous therapy. Acta Derm Venereol. 2020;100(14): adv00214.

21 Rigopoulos D, Gregoriou S, Makris M, Ioannides D. Efficacy of ustekinumab in nail psoriasis and improvement in nail-associated quality of life in a population treated with ustekinumab for cutaneous psoriasis: an open prospective unblinded study. Dermatology. 2011;223(4):325-9.

22 Reich K, Sullivan J, Arenberger P, Mrowietz U, Jazayeri S, Augustin M, et al. Effect of secukinumab on the clinical activity and disease burden of nail psoriasis: 32-week results from the randomized placebo-controlled TRANSFIGURE trial. Br J Dermatol. 2019 Nov;181(5):954-66.

23 Reich A, Szepietowski JC. Health-related quality of life in patients with nail disorders. Am J Clin Dermatol. 2011 Oct 1;12(5):31320.

24 Feldman SR. Psoriasis causes as much disability as other major medical diseases. J Am Acad Dermatol. 2020 Jan;82(1):256-7.

25 Menter A, Cordoro KM, Davis DMR, Kroshinsky D, Paller AS, Armstrong AW, et al. Joint American Academy of DermatologyNational Psoriasis Foundation guidelines of care for the management and treatment of psoriasis in pediatric patients. J Am Acad Dermatol. 2020 Jan;82(1):161-201.

26 Lewis-Jones MS, Finlay AY. The Children's Dermatology Life Quality Index (CDLQI): initial validation and practical use. Br J Dermatol. 1995 Jun;132(6):942-9.
The Impact of Nail Psoriasis and

Treatment on Quality of Life
Skin Appendage Disord 2021;7:83-89 DOI: $10.1159 / 000512688$ 